\title{
Repercusión de un protocolo de cuidados en la prevalencia de infección del orificio de salida del catéter de diálisis peritoneal
}

\author{
Magdalena Gándara Revuelta - Ana Begines Ramírez - Ma Teresa Sola García - Rosa Alonso Nates - José Luis \\ Cobo Sánchez - Carmen Higuera Roldan - Emilio Ibarguren Rodríguez
}

\section{Diplomados en Enfermería}

Servicio de Nefrología

Hospital Universitario Marqués de Valdecilla. Santander

\section{Sr. Director:}

Durante la década de los 60-70 del siglo pasado se desarrollaron las técnicas de diálisis, y con ello las posibilidades del peritoneo como membrana dializadora. El éxito de cualquier técnica de diálisis a largo plazo, se basa en disponer de un acceso permanente y seguro, en el caso de la diálisis peritoneal (DP), el catéter que nos sirve como acceso al peritoneo es un cuerpo extraño, y con frecuencia es fuente de infecciones, tanto cutáneas como del peritoneo.

Las infecciones del orificio de salida (OS) y del túnel del catéter en diálisis peritoneal son actualmente las causas más frecuentes de comorbilidad en este tipo de pacientes por lo que su prevención es el principal objetivo de enfermería desde la implantación del catéter y a lo largo de su permanencia. Los cuidados, tanto del personal sanitario como del propio paciente, son de vital importancia para disminuir la incidencia de infecciones del $\mathrm{OS}^{1,4}$.

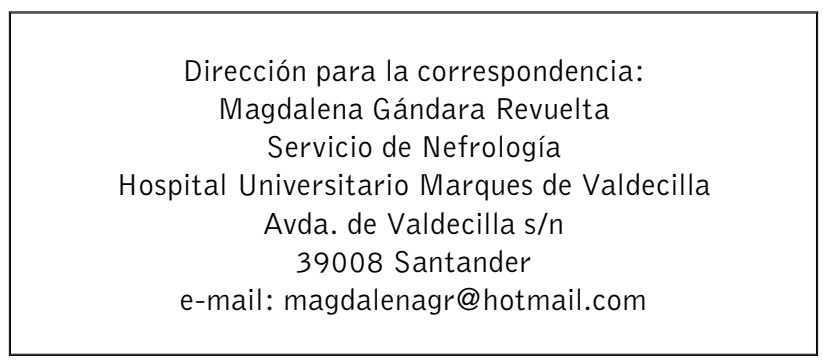

Una vez que el catéter está completamente curado y cicatrizado se debe educar al paciente en un correcto autocuidado.

Existen diferentes y variados protocolos para el cuidado de los 0S, recientes estudios muestran unos índices bajos de infección en los pacientes que solo utilizan el agua y jabón para el cuidado del OS con un secado exhaustivo ${ }^{5}$.

Con este trabajo nos planteamos los siguientes objetivos:

- Valorar la incidencia de infecciones del OS relacionados con el protocolo utilizado para su cuidado en nuestro hospital.

- Estudiar la incidencia de peritonitis relacionadas con la infección del 0S.

\section{Pacientes, material y métodos}

Hemos realizado un estudio descriptivo retrospectivo de toda la población de pacientes con catéter peritoneal durarte los últimos tres años, para lo que hemos revisado los registros de enfermería en las historias de todos los pacientes portadores de catéter peritoneal desde Enero del 2007 a Diciembre del 2009.

Se estudiaron las variables edad, sexo, tiempo de permanencia del catéter peritoneal, OS cubierto o descu- 
bierto, incidencia de diabetes e incidencia de infecciones relacionadas con el catéter de diálisis peritoneal.

La valoración de los orificios de salida fue realizada por 2 enfermeras encargadas de la Diálisis peritoneal, utilizando la clasificación de Twardowski ${ }^{6}$. Se realizó su valoración cuando el paciente acudía a la consulta programada cada mes. De aquellos OS que presentaban signos o criterios de infección se recogieron cultivos.

En todos los pacientes se ha seguido el protocolo de cuidados del OS del catéter peritoneal de nuestro hospital, que consiste en:

- Las primeras curas se realizaran por la enfermera de Diálisis peritoneal a las $48 \mathrm{~h}$, a los 5 y 10 días tras la implantación del catéter, utilizando siempre una técnica estéril: limpieza con suero salino y povidona iodada, dejando cubierto hasta la siguiente cura.

- A los 10 días de la intervención se retiran los puntos de la herida quirúrgica y a partir de este día el paciente se puede duchar sujetando el catéter para que no cuelgue del orificio, evitando tirones.

- Educamos al paciente en el cuidado de su OS aconsejando ducha diaria.

- Para la ducha se les indica que tras retirar todos los apósitos, se jabonen todo el cuerpo y el orificio de salida sin esponjas, se aclaren bien todo el cuerpo y por último el OS; secando con toalla todo el cuerpo, excepto el OS que se hará secando bien con gasas estériles.

- Es opcional taparlo o dejarlo al aire. Si lo tapa se pondrá una gasa debajo sin doblar y otra encima y luego lo cubrirá con esparadrapo. Sí lo deja al aire es conveniente sujetar el catéter con un esparadrapo para no dar tirones o en su defecto sujetarlo con la ropa interior.

- Por último evitar el uso de cinturón o gomas en la zona del orificio que puedan dañarlo o irritarlo.

\section{Resultados}

Hemos estudiado 118 pacientes, de los que el 71,2\% (84 pacientes) eran varones y el 28,8\% (34 pacientes) mujeres, con una edad media de 62 años y un rango de 15 a 87 años en el momento de la colocación del catéter.

El tiempo de permanencia del catéter fue de 28,7 meses (rango 1-128 meses), presentando infección del oS un 16,1\% (19 pacientes). De éstos, 14 pacientes llevaban el OS cubierto, y 5 pacientes lo llevaban descubierto.

La descripción de la evolución de los orificios queda representada en el gráfico 1 . Los microorganismos responsables de las infecciones del $0 \mathrm{~S}$ se presentan en el gráfico 2. En cinco pacientes no se aislaron gérmenes y en 9 episodios el agente causal fue el Staphylococcus Aureus.

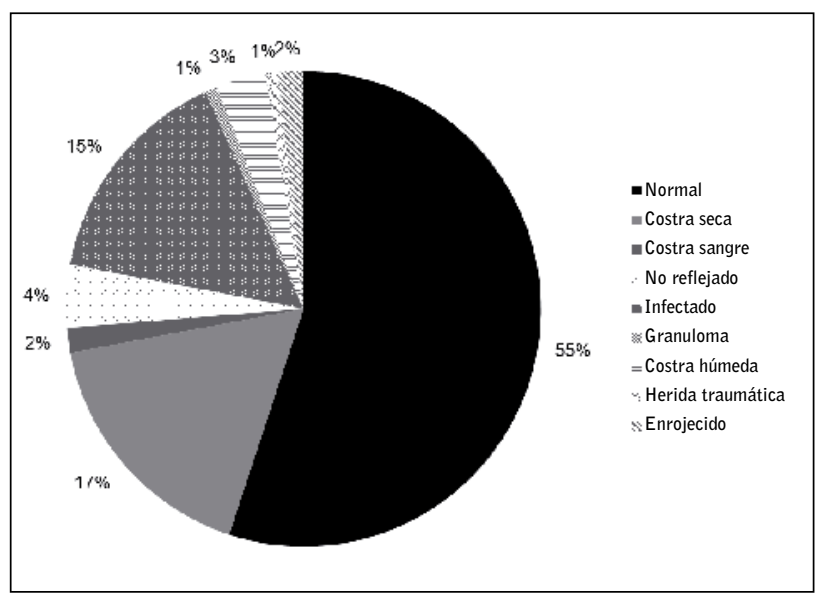

Gráfico 1. Evolución de los orificios de salida según criterios de clasificación de Twardowski

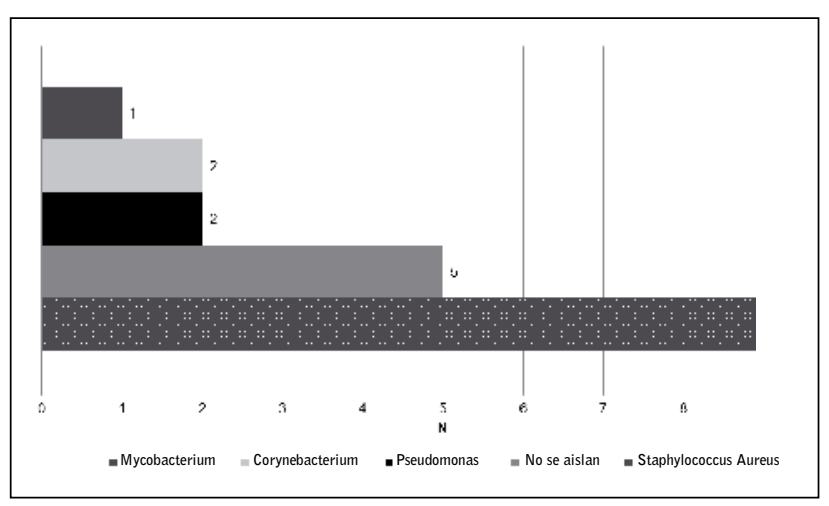

Gráfico 2. Gérmenes responsables de la infección del OS en los pacientes estudiados 
En los pacientes que presentaron un episodio de infección del OS estudiamos la existencia de peritonitis y si estaba relacionada con el proceso del orificio 0 no. Encontramos que el $57 \%$ no había tenido ningún episodio de peritonitis, el $21 \%$ (4 pacientes) tuvieron una peritonitis que no se pudo relacionar con el $0 S$, un $21 \%$ (4 pacientes) no pudo continuar en la técnica ya que fue necesario retirar el catéter, y solo un paciente tuvo una peritonitis en relación con la infección del OS que obligó a un recambio del catéter $(5,2 \%)$.

Fue necesario retirar 5 catéteres (4,2\%) de los 118 pacientes estudiados:

- Un paciente que presentaba infección del OS se retiró por presentar un hidrotórax y se transfirió a hemodiálisis.

- Tres pacientes presentaron infección del OS relacionada con la herida quirúrgica de los cuales uno era diabético, otro trasplantado renal, diabético y con deterioro de la función renal, todos ellos fueron retirados del programa de Diálisis Peritoneal.

Sólo observamos un caso de una peritonitis relacionada con la infección del OS. Este catéter tenía una vida de 8 años. Presentó un episodio de infección del túnel subcutáneo con exudado que obligó a su retirada. Se colocó un nuevo catéter y el paciente continúa en la actualidad en Diálisis Peritoneal sin más complicaciones.

\section{Discusión}

El objetivo fundamental de los cuidados del OS es prevenir las complicaciones, sobre todo las infecciosas y traumáticas para lo que es fundamental la limpieza frecuente y una fijación correcta del catéter para evitar lesiones en el $\mathrm{OS}$ y el túnel subcutáneo. En nuestro protocolo utilizamos la limpieza con agua y jabón, presentando una incidencia de infección del OS del $16 \%$. Gómez Castilla y cols 5 , en un reciente estudio, estudiaron el comportamiento de 306 pacientes procedentes de distintos hospitales andaluces, que utilizaban distintos procedimientos para la limpieza del $0 \mathrm{~S}$. Encontraron que los más bajos índices de infección se presentaban en aquellos pacientes que sólo utilizaban agua y jabón así como en los que usaban povidona yodada, frente a los que realizaban la cura sólo con suero, procedimiento combinado u otro procedimiento. También observaron que las infecciones del OS por gram positivo aparecían con mayor frecuencia en aquellos pacientes que no retiraban el apósito durante la ducha. Lima y cols ${ }^{7} \sin$ embargo, defienden que hay que proteger el $0 \mathrm{~S}$ del agua doméstica, cubriéndolo durante la ducha con apósitos oclusivos y limpiándolo con agua hervida.

En nuestra opinión, lo fundamental para prevenir la infección del OS es realizar, además de una higiene adecuada del mismo, un correcto secado de la zona tras su limpieza. Gómez Castilla y cols ${ }^{5}$ encontraron un aumento de infecciones por pseudomona cuando el paciente no procedía al secado del OS con secador. A nuestro modo de ver, secar la zona con gasas estériles de forma minuciosa previene la aparición de infecciones, ya que nuestros resultados sólo muestran dos infecciones por pseudomona.

La baja incidencia de peritonitis relacionada con infección del OS mejora los resultados de otras series ${ }^{5}$, junto con la baja incidencia de retirada del catéter (una de ellas relacionada con problemas mecánicos y no por infección), pone de manifiesto que los dos cuidados destacados (limpieza con agua y jabón y un secado óptimo) disminuyen las complicaciones derivadas de la infección del 0S.

Después de la completa cicatrización del catéter los cuidados frecuentes del $0 S$ deben estar basados en una correcta enseñanza al paciente de su responsabilidad en el cuidado del mismo. Un aseo correcto con agua y jabón, y lo más importante, la ausencia de humedad en el OS, secando bien con gasas estériles, previenen la aparición de infecciones del OS.

Es imprescindible recordar siempre a los pacientes portadores de un catéter de Diálisis Peritoneal que deben evitar todas aquellas maniobras que supongan tirones o torsiones del mismo sobre el OS, debiendo estar sujeto a la piel o con la ropa interior. Estas recomendaciones por sí solas han mejorado la duración de los catéteres y evitado o disminuido el número de infecciones.

Es una labor de enfermería trasmitir al paciente la importancia que estos cuidados tienen sobre su bienestar y en el mantenimiento de la técnica. 


\section{Bibliografía}

1. Rietmann A, Casal MC. Guía de Práctica clínica de Diálisis Peritoneal de la EDTNA/ERCA. Luzern: EDTNA/ERCA; 2009.

2. Muñoz Poyato J. Manual de protocolos y procedimientos de actuación de enfermería nefrológica SEDEN. Madrid: Entheos;2001.

3. Coronel F, Montenegro J, Selga R, Celadilla 0, Tejuca M (Eds). Manual Práctico de Diálisis Peritoneal (SEDEN - SEN). Badalona: Editorial Atrium; 2005.

4. Arrieta J, Bajo MA, Caravaca F, Coronel F, García Pérez $\mathrm{H}$, González Parra E y cols. Guías de práctica clínica en Diálisis Peritoneal. Nefrología 2006;26 (Supl 4).
5. Gómez Castilla AC, Martín Espejo JL, Trujillo Campos C, Laguillo de Castro A, Bejuca Marenco M, López Bermúdez $\mathrm{E}$ y cols. Comportamiento del orificio de implantación del catéter en pacientes en diálisis peritoneal en relación a los cuidados. Rev Soc Esp Enferm Nefrol 2007; 10 (4): 263-269.

6. Twardowski ZJ, Prowant BF. Classification of normal and diseased exit-sites. Perit Dial Int 1996;16 Suppl 3:S32-S50.

7. Lima Comas C, Alcantud García MJ, Gámez Ráez N, Pastor Palenzuela A, Gómez Marqués G, Morey Molina A. Los apósitos oclusivos en la ducha diaria ¿son capaces de reducir las infecciones del orificio de salida del catéter peritoneal? Rev Soc Esp Enferm Nefrol 2007; 10 (2): 144-148. 